\title{
EDITORIAL
}

For reprint orders, please contact: reprints@futuremedicine.com

\section{Implementing lung cancer screening: a checklist}

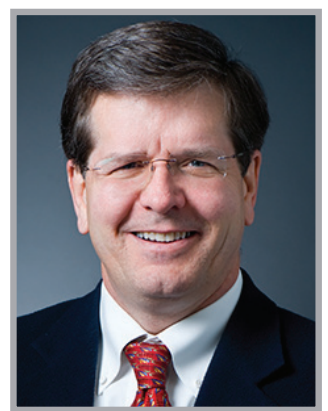

James L Mulshine ${ }^{* 1}$

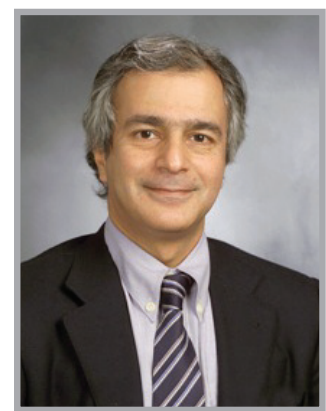

Nasser Altorki
"As lung cancer screening matures, the process of implementing screening will become much more familiar but, for now, there are formidable challenges in initiating and sustaining this service."
Based on the results of the NLST [1], the US Preventative Services Task Force (USPSTF) has affirmed its recommendation regarding the value of low-dose computed tomography $(\mathrm{CT})$ scans in finding early lung cancer [2]. The next steps entail ensuring provisions for appropriate reimbursement followed by national implementation of this screening service. From a clinical perspective, low-dose CT management is quite different from existing management strategies of clinically detected lung cancer. Therefore the transition to this new management approach may be challenging. Inherent in the emergence of lung cancer screening as a recommended service is that the optimal organizational structure to provide this service is not fully defined. Recent articles have outlined how risk stratification and diagnostic workups as well as surgical interventions can be refined to further improve screening outcomes $[1,3,4]$.

For a new multidisciplinary team beginning to provide lung cancer screening care what is the best strategy for rapidly implementing the delivery of safe, quality screening service? Recently, the 'checklist' approach has been advocated as a mechanism that decreases harm and enhances the favorable outcomes of complex pathways of care [101]. In this editorial, we will use a checklist as a metaphor to propose how to facilitate the evolution of high-quality lung cancer screening.

So what is the checklist to ensure prudent attention to core issues as institutions consider implementation of a lung cancer screening program (Box 1). A critical initial factor is to coordinate this process with the appropriate institutional administrators. An institutional commitment to finance the necessary infrastructure should include space allocation and the provision of the informatic and ancillary staff support required for a successful inception of a screening program. The USPSTF has finalize their recommendation for lung cancer screening services, but a number of private insurers have not yet added this service to their coverage provisions. Therefore, for the intermediate term, such reimbursement issues need to be addressed proactively, so that appropriate financial provisions can be arranged so that individuals that require further

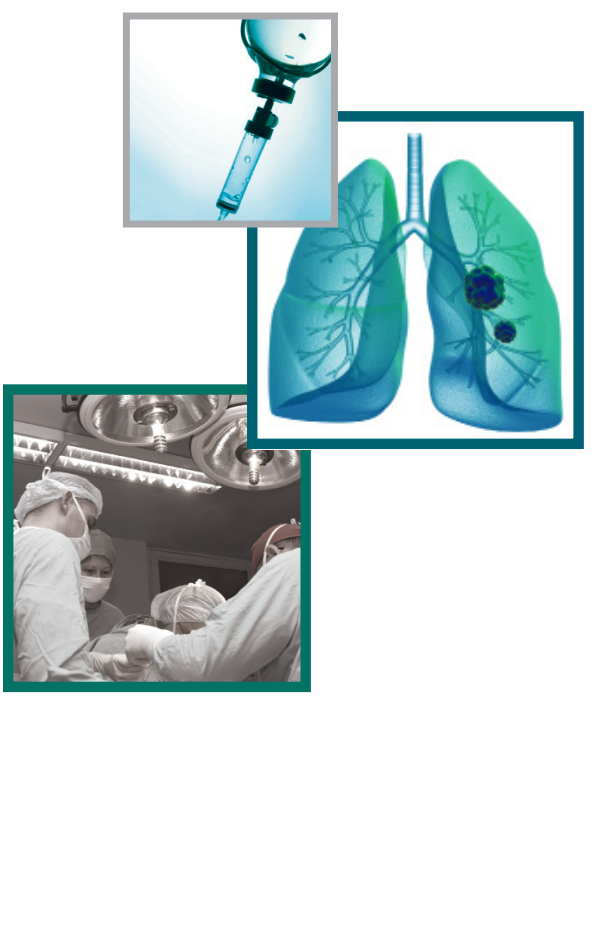

\footnotetext{
"The challenge is to ensure close follow-up as recommended for suspicious pulmonary nodules, for early awareness of local regional recurrences in surgical patients

or for the evolution of new primary lung cancers."
}

'Rush University, 1735 West Harrison Street, Suite 206, Chicago, IL 60612, USA

${ }^{2}$ Weill Medical College, Cornell University, 525 East 68th Street, F2212, New York, NY 10021, USA

*Author for correspondence: jmulshin@rush.edu

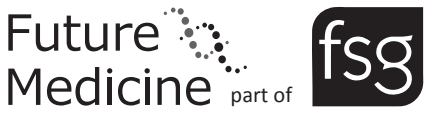




\section{Box 1. Checklist for institutional screening implementation.}

- Administrative issues with providing best practice lung cancer screening

- Communication plan

- Integration of tobacco cessation measures

- Involvement of qualified interdisciplinary team

- Eligibility and informed consent plan

- Providing evidence-based diagnostic work-up algorithm

- Provisions for robust quantitative imaging and image processing

- Provisions for optimized surgical management

- Provisions for timely and reliable follow-up process

care for the screening evaluation have access to indicated care.

Once institutional support is secured the next item in the 'checklist' requires the interdisciplinary collaboration of clinicians, radiologists, pulmonologists, thoracic surgeons and pathologists who collectively develop the appropriate management strategies and assemble the array of clinical services required for high-quality screening services. These potentially include confirmation of eligibility, informed consent discussion, quantitative low-dose CT imaging, diagnostic work-up and various interventional and surgical services, as well as ensuring timely follow-up care. Another issue to consider is that many people considering lung cancer screening will be current smokers. There is already a consensus that the best practice for lung cancer screening includes providing smoking cessation advice [1]. This measure is critical since continued smoking is associated with continued increase in the risk of developing lung cancer, as well as the significant risk of premature death due to tobaccoinduced cardiovascular or pulmonary disease. Therefore, the obligation of a facility offering CT screening service is to include routine access to tobacco cessation counseling [1]. Since many people undergoing screening will continue to smoke this is a great opportunity to explore more effective approaches to smoking cessation and enhance the public health benefit of this encounter [5].

An important item on the checklist is the constant monitoring of the quality and outcomes of each delivered service in a manner that constantly re-examines, refines and streamlines the entire process. This may additionally include participation and compliance with smoking cessation initiatives. The advocacy group, the Lung Cancer Alliance has proposed a consortial environment they term the National Framework to encourage screening institutions to cooperate in voluntarily committing to deliver best-practice approach to lung cancer screening care [6]. This includes systematic reporting of their outcomes while providing screening services.

Recent publications from the USPSTF, American Cancer Society and National Comprehensive Cancer Network have attempted to synthesize the state of the rapidly evolving evidence to support low-dose CT screening $[1,6,7]$. These information sources provide an important resource for an objective discussion of the benefits and harms of low-dose CT screening with high risk for lung cancer individuals considering participation in lung cancer screening. While this reference list is by no means exhaustive, best practice suggests that individuals considering screening participation should be provided with a clear and objective discussion about the merits of their screening participation.

Given the alarmingly high false-positive rate reported by the NLST, the importance of a well-conceived and strictly-adhered-to diagnostic protocol cannot be overemphasized. A recent article from International-Early Lung Cancer Action Project that analyzed the efficiency of their diagnostic work-up of screen-identified suspicious lung nodules suggested that falsepositive work-ups could be greatly reduced if nodule work-up was restricted to pulmonary nodules exceeding $8 \mathrm{~mm}$ in diameter [4]. The International-Early Lung Cancer Action Project group has been extensively involved in lung cancer screening research and as part of their master protocol have developed an important on-boarding process involving double reading of the first hundred screening CT images performed at a new screening site [102]. After reaching this number a conference call is setup to review the discrepancies in the reading interpretation [103]. Discrepancies between the coordinating center and the new site are still monitored at defined intervals so that there is closer standardization with the subsequent screening management [1]. The approach to diagnostic work-up is a dynamic area due to efforts to minimize unnecessary invasive diagnostic procedures, accordingly groups such as National Comprehensive Cancer Network have regularly updated their guidelines to incorporate evolving evidence [6].

A major challenge with screening implementation is ensuring that the screening process 
embeds quality imaging processes. The Radiological Society of North America has invested considerable effort in to defining a process to achieve such a result. A committee called the Quantitative Imaging Biomarker Alliance has worked to define the best approach to reproducible image acquisition and then measuring volumetric changes in suspicious nodules for lung cancer screening [8]. These provisions are designed to minimize measurement errors that could lead to inappropriate clinical management. We have been working with a variety of collaborators to thoughtfully integrate these quantitative imaging approaches into efficient and effective clinical care processes [9].

Some have suggested that surgical services inappropriately applied can substantially alter the risk-benefit formula underlying successful screening. Mitigating that risk requires early involvement of thoracic surgeons in every step of the screening process including conception of the diagnostic protocol and the development of minimally invasive diagnostic and localization strategies. Therapeutic surgical intervention should also emphasize minimally invasive video-assisted or robotic approaches that have been shown to be associated with significantly lower morbidity and duration of hospitalization. Finally, it seems logical to propose that detection of smaller tumors in the context of screening demands revisiting the extent of parenchymal resection conventionally applied to clinically detected disease. For example, a recent paper by the International-Early Lung Cancer Action Project surgical investigators showed that 10-year survival in screen-detected clinical stage $1 \mathrm{~A}$ non-small-cell lung cancer was essentially identical after either lobar or sublobar resection [10]. There are currently two large ongoing randomized trials in Japan and North America addressing the validity of sublobar resection in peripheral clinical stage $1 \mathrm{~A}$ non-small-cell lung cancer [11,104].

As lung cancer screening matures, the process of implementing screening will become much more familiar but, for now, there are formidable challenges in initiating and sustaining this service. It seems reasonable that screening activities be, at least initially, limited to major institutions that posses the resources and the necessary clinical services to effectively and safely deliver the service. A close collaboration must be developed with primary care providers to ensure timely and appropriate follow-up care is delivered. The challenge is to ensure close follow-up as recommended for suspicious pulmonary nodules, for early awareness of local regional recurrences in surgical patients or for the evolution of new primary lung cancers.

Finally, we again emphasize how a lung cancer screening program is an excellent platform to continually make advances, one step at a time, in the battle against smoking.

\section{Financial \& competing interests disclosure}

The authors have no relevant affliations or financial involvement with any organization or entity with a financial interest in or financial conflict with the subject matter or materials discussed in the manuscript. This includes employment, consultancies, honoraria, stock ownership or options, expert testimony, grants or patents received or pending, or royalties.

No writing assistance was utilized in the production of this manuscript.

\section{References}

1 Aberle DR, DeMello S, Berg CD et al. Results of the two incidence screenings in the National Lung Screening Trial. N. Engl. J. Med.369(10), 920-931 (2013).

2 Humphrey LL, Deffebach M, Pappas M et al. Screening for lung cancer with low-dose computed tomography: a systematic review to update the US Preventive Services Task Force recommendation. Ann. Intern. Med. 159(6), 411-420 (2013).

3 Tammemagi MC, Katki HA, Hocking WG et al. Selection criteria for lung-cancer screening. N. Engl. J. Med. 368(8), 728-736 (2013).

4 Henschke CI. Definition of a positive test result in computed tomography screening for lung cancer: a cohort study. Ann. Intern. Med. 158(4), 246-252 (2013).

5 Pyenson B, Sander M, Jiang Y, Kahn H, Mulshine J. An actuarial analysis shows that offering lung cancer screening as an insurance benefit would save lives at relatively low cost. Health Aff. 31(4), 770-779 (2012).

6 Wood D, Eapen G, Ettinger D et al. Lung cancer screening. J. Natl Compr. Cancer Network 10(2), 240-265 (2012).

7 Wender R, Fontham E, Barrera E et al. American cancer society lung cancer screening guidelines. CA Cancer J. Clin. 63(2), 107-117 (2013).

8 Buckler AJ, Mulshine JL, Gottlieb R, Zhao B, Mozley PD, Schwartz L. The use of volumetric $\mathrm{CT}$ as an imaging biomarker in lung cancer. Acad. Radiol. 17(1), 100-106 (2010).

9 Mulshine JL, Avila R, Yankelevitz D et al. Application of high-resolution ct imaging data to lung cancer drug development: measuring progress: workshop IX. J. Thorac. Oncol. 8(11), 1352-1355 (2013).

10 Altorki NK, Yip R, Hanaoka T et al. Sub-lobar resection is equivalent to lobectomy for clinical stage 1A lung cancer in solid nodules. J. Thorac. Cardiovasc. Surg. doi:10.1016/j.jtcvs.2013.09.065 (2013) (Epub ahead of print).

11 Nakamura K, Saji H, Nakajima R et al. A Phase III randomized trial of lobectomy versus limited resection for small-sized peripheral non-small cell lung cancer 
EDITORIAL Mulshine \& Altorki

(JCOG0802/WJOG4607L). Jpn J. Clin. Oncol. 40(3), 271-274 (2010).

\section{- Websites}

101 WHO. Patient safety checklists. www.who.int/patientsafety/implementation/ checklists/en

(Accessed 13 January 2014)
102 Henschke CI. International early lung cancer action program: enrollment and screening protocol.

http://ielcap.org/sites/default/files/ielcap.pdf (Accessed 13 January 2014)

103 Rights and expectations for excellence in lung cancer screening and continuum of care. www.screenforlungcancer.org/nationalframework

(Accessed 17 September 2012)

104 Alliance/CALGB 140503 trial. www.calgb.org/Public/meetings/ presentations/2007/cra_ws/03-140501-

Altorki062007.pdf

(Accessed 13 January 2014) 